\title{
Hypoxia Improves Endurance Performance by Enhancing Short Chain Fatty Acids Production via Gut Microbiota Remodeling
}

\author{
Li Huang, Tianyou Li, Min Zhou, Mengyan Deng, Lidong Zhang, Long Yi, Jundong Zhu, \\ Xiaohui Zhu* and Mantian Mi*
}

Chongqing Key Laboratory of Nutrition and Food Safety, Research Center for Nutrition and Food Safety, Chongqing Medical Nutrition Research Center, Institute of Military Preventive Medicine, Third Military Medical University, Chongqing, China

OPEN ACCESS

Edited by:

llias Lagkouvardos,

Technical University of Munich,

Germany

Reviewed by:

Hannah Harris,

Quadram Institute, United Kingdom

Seungha Kang,

University of Queensland, Australia

*Correspondence:

Xiaohui Zhu

zhu_xh@yeah.net

Mantian Mi

mi_mantian@sina.com

Specialty section: This article was submitted to

Microbial Symbioses,

a section of the journal

Frontiers in Microbiology

Received: 23 November 2021 Accepted: 28 December 2021

Published: 07 February 2022

Citation:

Huang L, Li T, Zhou M, Deng M, Zhang L, Yi L, Zhu J, Zhu X and Mi M (2022) Hypoxia Improves Endurance

Performance by Enhancing Short Chain Fatty Acids Production via Gut

Microbiota Remodeling.

Front. Microbiol. 12:820691. doi: 10.3389/fmicb.2021.820691
Hypoxia environment has been widely used to promote exercise capacity. However, the underlying mechanisms still need to be further elucidated. In this study, mice were exposed to the normoxia environment $\left(21 \% \mathrm{O}_{2}\right)$ or hypoxia environment $(16.4 \%$ $\mathrm{O}_{2}$ ) for 4 weeks. Hypoxia-induced gut microbiota remodeling characterized by the increased abundance of Akkermansia and Bacteroidetes genera, and their related short-chain fatty acids (SCFAs) production. It was observed that hypoxia markedly improved endurance by significantly prolonging the exhaustive running time, promoting mitochondrial biogenesis, and ameliorating exercise fatigue biochemical parameters, including urea nitrogen, creatine kinase, and lactic acid, which were correlated with the concentrations of SCFAs. Additionally, the antibiotics experiment partially inhibited hypoxia-induced mitochondrial synthesis. The microbiota transplantation experiment demonstrated that the enhancement of endurance capacity induced by hypoxia was transferable, indicating that the beneficial effects of hypoxia on exercise performance were partly dependent on the gut microbiota. We further identified that acetate and butyrate, but not propionate, stimulated mitochondrial biogenesis and promoted endurance performance. Our results suggested that hypoxia exposure promoted endurance capacity partially by the increased production of SCFAs derived from gut microbiota remodeling.

Keywords: endurance performance, gut microbiota, hypoxia, short chain fatty acids, mitochondrial biogenesis

\section{INTRODUCTION}

There are many benefits of hypoxia, such as helping weight loss, maintaining cardio-metabolic health (Kayser and Verges, 2013; Woolcott et al., 2015; Hobbins et al., 2017), as well as improving exercise performance (Girard et al., 2013a,b; Wang et al., 2019). Exercise training under hypoxic conditions has been widely accepted as a mean to enhance athletic endurance ability in the past few decades (Girard et al., 2013a; Millet and Brocherie, 2020). The predominant mechanisms of hypoxic training on improving sports performance focused on hematological factors such as increased red blood cell (RBC) counts and high hemoglobin (HGB) concentrations, as well as non-hematological factors including running economy, lactate threshold, mitochondrial gene expression, and enhanced muscle buffering capacity (Saunders et al., 2013; Mujika et al., 2019). However, further researches are needed to clarify the other mechanisms underlying hypoxia increasing exercise performance. 
The gut microbiota and its hosts maintain a mutually beneficial relationship and dynamic coexistence (Fan and Pedersen, 2021). Recent evidence indicated that the gut microbiota plays a significant role in exercise performance (Lahiri et al., 2019; Mailing et al., 2019; Nay et al., 2019; Scheiman et al., 2019). Compared with specific pathogen-free mice, germ-free mice showed skeletal muscle atrophy and lower endurance capacity, while recovering the gut microbiota of germfree mice resulted in improved endurance performance and skeletal muscle mass increasing (Hsu et al., 2015; Huang et al., 2019; Lahiri et al., 2019). Veillonella, enriched in marathon runners, could metabolize lactate into acetate and propionate and converse inorganic nitrate to nitric oxide and related nitrogen oxides, thus improving exercise performance (Scheiman et al., 2019; Lundberg et al., 2021). Moreover, short-chain fatty acids (SCFAs) produced by the gut microbiota are now recognized as the potential regulators of skeletal muscle metabolism and function to enhance exercise performance (Okamoto et al., 2019; Frampton et al., 2020; Hu et al., 2020; Bongiovanni et al., 2021). These findings indicated that the gut microbiota might improve exercise performance through its derived metabolites participating in host metabolism.

In recent years, the effects of hypoxia on the gut microbiota attracted much attention (Mazel, 2019; Han et al., 2021). Short-term or chronic exposure to hypoxia can influence the composition and diversity of the gut microbiota ( $\mathrm{Li}$ and Zhao, 2015; Karl et al., 2018; Suzuki et al., 2019; Jia et al., 2020), thus helping the hosts to adapt to the environment (Li et al., 2016, 2018, 2019; Sun et al., 2019; Ma et al., 2021). Current findings indicated that hypoxia exposure leads to intestinal hypoxia, thus promoting the growth of anaerobic bacteria (Suzuki et al., 2019; Jia et al., 2020). However, the interventions considerably varied with regards to the hypoxia model such as exposure time and oxygen concentration, and to the confounders such as exercise or diet. Thus, the influences of hypoxia exposure on the gut microbiota are still controversial (Li and Zhao, 2015; Karl et al., 2018; Suzuki et al., 2019; Han et al., 2020, 2021; Jia et al., 2020).

Hence, we aimed to investigate the mechanisms underlying hypoxia exposure enhancing endurance performance. Interestingly, we found that hypoxia significantly improved endurance performance by increasing the abundance of the Akkermansia and Bacteroides genus. Further results revealed that the SCFAs produced by the gut microbiota enhanced mitochondrial biogenesis, thus improving endurance capacity. Our results suggested that hypoxia promotes exercise performance through the regulation of the gut microbiota-SCFAs axis.

\section{MATERIALS AND METHODS}

\section{Animals}

Healthy male C57BL/6J mice (6-8 weeks old) were acquired from the Animal Experimental Center of Third Military Medical University (Chongqing, China). Animals were housed under a 12-h light/dark cycle with ad libitum access to food and water. During the experiments, body weight was recorded weekly. Before the experiments, mice were placed in a motorized treadmill (SANS Biological Technology, China) and ran at 15 $\mathrm{m} / \mathrm{min}$ speed for $10 \mathrm{~min}$ once a day for 5 days as acclimatized training. At the end of the experiments, mice ran at the speed of $25 \mathrm{~m} / \mathrm{min}$ at normobaric normoxia until exhaustion as endurance test. In the acclimatized training and the endurance test, the acceleration was $5 \mathrm{~m} / \mathrm{min}^{2}$, and timing started when the speed reached 15 and $25 \mathrm{~m} / \mathrm{min}$, respectively. The current of electrode slice was set to $2 \mathrm{~mA}$ to prod mice to keep running. In the endurance test, exhaustion was defined as the inability to run for $20 \mathrm{~s}$ under the prodded electrode slice, as we described before (Hou et al., 2020). After mice exhaustion, blood samples were taken from the tail vein to measure blood glucose and lactic acid with the OneTouch blood glucose meter (LifeScan, United States) and Lactate Scout (EKF, Germany), respectively. Then mice were sacrificed immediately. Soleus and gastrocnemius muscle mass were examined by an electronic scale and stored at $-80^{\circ} \mathrm{C}$. The Animal Care and Use Committee of the Third Military Medical University approved all the animal experiments.

\section{Experimental Design}

The experimental design shown in Supplementary Figure 1.

\section{Animal Experiment 1}

To determine the effects of hypoxia on exercise performance and gut microbiota, mice were randomly divided into two groups: normobaric normoxia (Normoxia; 21.0\% $\mathrm{O}_{2}$ ) and normobaric hypoxia (Hypoxia; $\left.16.4 \% \mathrm{O}_{2}\right)(n=8 /$ group). Grip strength and hematological parameters were measured at the different time points of 0,2 , and $4 \mathrm{w}$. Stool samples from both groups were collected at the end of treatment (Supplementary Figure 1A).

\section{Animal Experiment 2}

To identify the role of gut microbiota in hypoxia improving exercise intolerance, we performed antibiotic treatment and fecal microbiota transplantation (FMT) experiments.

a. For the antibiotic treatment experiment, mice were randomly divided into two groups ( $n=9$ /group). One group (Hypoxia) was exposed to a hypoxia environment for 4 weeks. The other group (Hypoxia + Abx) was exposed to a hypoxia environment accompanied by $200 \mu \mathrm{L}$ antibiotic cocktail (Abx) ( $1 \mathrm{~g} / \mathrm{L}$ ampicillin, $1 \mathrm{~g} / \mathrm{L}$ metronidazole, $1 \mathrm{~g} / \mathrm{L}$ neomycin, $0.5 \mathrm{~g} / \mathrm{L}$ vancomycin) by gavage for 7 days to clear gut microbiota. During the remaining 3 weeks, mice were treated with $200 \mu \mathrm{L}$ Abx every other day to keep the flora eliminated (Supplementary Figure 1B; Hui et al., 2020). Mice treated with Abx did not show any illness or distress.

b. Stool samples collected from animal experiment 1 were dissolved in PBS $(20 \mathrm{mg} / \mathrm{mL})$, shaken for $3 \mathrm{~min}$, and centrifuged for $3 \mathrm{~min}$. The supernatant was collected and stored at $-80^{\circ} \mathrm{C}$. The receipt mice were treated with $200 \mu \mathrm{L}$ Abx by gavage for 1 week. Then, mice were treated with $100 \mu \mathrm{L}$ of the supernatant once daily for seven consecutive days in the first week of colonization. During the remaining 3 weeks, fecal slurries were introduced every other day to reinforce colonization ( $n=9 /$ group) (Supplementary Figure 1C). 


\section{Animal Experiment 3}

To identify the important role of SCFAs in hypoxia improving exercise endurance, mice received $150 \mathrm{mM}$ acetate (Sigma, United States), propionate (Sigma, United States), butyrate (Sigma, United States) or $\mathrm{NaCl}$ (vehicle) in the drinking water for 4 weeks as reported before ( $n=9$ /group) (Supplementary Figure 1D; Smith et al., 2013; Fachi et al., 2019).

\section{Hypoxia Treatment}

An $8 \mathrm{~mm}$ thick acrylic cabin $(180 \times 50 \times 50 \mathrm{~cm})$ was used to create a normobaric hypoxia environment. The normobaric hypoxia cabin was designed by Professor Xiaohui Zhu, and produced by TOW-INT Tech (China). Environmental hypoxia was generated by adjusting the relative concentrations of oxygen and nitrogen in the input gas mixture. With constant monitoring, continuous gas flow and $\mathrm{CO}_{2}$ absorption by $\mathrm{Ca}(\mathrm{OH})_{2}$ within the hypoxic cabin maintained $\mathrm{CO}_{2}$ levels below $0.5 \%$. Adjust the $\mathrm{O}_{2}$ concentration in the cabin to $16.4 \%$ within $30 \mathrm{~min}$, equivalent to an altitude of $2,000 \mathrm{~m}$. A controlled environment $\left(21 \% \mathrm{O}_{2}\right)$ was created with an identical cabin setup. During the experiment, the temperature was maintained at $23 \pm 2{ }^{\circ} \mathrm{C}$, and the humidity was $65 \pm 5 \%$. Changing food, sawdust, and $\mathrm{Ca}(\mathrm{OH})_{2}$ every day from 9:00 to 10:00 at normoxia environment.

\section{Grip Strength Test}

Grip strength was measured by a grid-connected to the grip strength meter (Ugo Basile, Germany). The mice underwent three tests, with at least $10 \mathrm{~min}$ recovery time between tests. The mean measure of maximal force (in grams) recorded was used as the grip strength.

\section{Hematological Parameters Measurement}

Blood was collected from the tail veins of mice and diluted in $1 \%$ EDTA solution. Red blood cell (RBC) counts and hemoglobin (HGB) concentration were measured automatically by a hematology analyzer (XT-1800i/2000IV; Sysmex, Kobe, Japan).

\section{Biochemical Parameters Measurement}

Serum was obtained from blood samples in mice, and biochemical profiles including creatine kinase $(\mathrm{CK})$ and urea nitrogen (BUN) were measured with commercial kits (Solarbio, China). Hepatic glycogen was determined using Glycogen Detection Kit (Solarbio, China) according to the manufacturer's instruction. All the experiments were tested at least three times.

\section{Transmission Electron Microscopy Observation}

Mitochondrial morphology and number in the gastrocnemius muscle were analyzed by TEM images. Briefly, gastrocnemius was treated and visualized on JEM-1400 microscope (Jeol, Japan) as we described before (Liu et al., 2020). Three animals per group were and ten images per mouse were used for the quantitative analysis of mitochondria. The number of mitochondria per section of intermyofibrillar mitochondria was quantified. The number of mitochondria was counted on micrographs as previously described (Liu et al., 2020).

\section{Sequencing of the Gut Microbiota}

The bacterial genomic DNA from stool samples was extracted using a DNA Stool kit (TianGen, China). The DNA sequence was amplified using barcoded $\mathrm{V} 3-\mathrm{V} 4$ region primers targeting the bacterial 16S rRNA gene, sequenced on the Illumina MiSeq platform (Illumina, San Diego, CA, United States). Analyses of generated and demultiplexed sequences were performed using the QIIME software (v.1.8.0) (Caporaso et al., 2010). Operational taxonomic units (OTUs) with 97\% similarity cutoff were clustered using UPARSE (v.7.1) (Edgar, 2013), and chimeric sequences were identified and removed. The $\alpha$-diversity indexes were calculated by Mothur (v.1.30.1) (Schloss et al., 2009). Principal coordinate analysis (PCoA) based on the unweighted UniFrac distance was performed using the QIIME software. The discriminated taxa of the gut microbiota profiles in different groups were identified using the R and STAMP (v.2.0.0) (Segata et al., 2011). To determine the microbiota-based biomarkers, the Linear Discriminant Analysis Effect Size (LEfSe) method was used, which indicates the taxa that contribute to the uniqueness of the corresponding groups at a linear discriminant analysis (LDA) score of $>2.0$ (false discover rate $($ FDR $)<0.5)$.

\section{Measurement of Cecal Contents Short-Chain Fatty Acids}

Fatty acid analysis was conducted using an Agilent $6890 \mathrm{~N}$ GC system (Agilent Technologies, PA, United States) and performed as previously described (Zhao et al., 2006; Kang et al., 2017). Briefly, cecal contents from each mouse were weighed and homogenized in $1 \mathrm{~mL}$ deionized water for $3 \mathrm{~min}$. The $\mathrm{pH}$ value of the suspension was adjusted to $2-3$, and the suspension was subsequently transferred into a polypropylene tube and centrifuged for $20 \mathrm{~min}$ at 3,000 g, yielding a clear supernatant. 2-ethylbutyric acid (TEBA) was used as the internal standard and added into the supernatant at a final concentration of $1 \mathrm{mM}$.

\section{Quantitative RT-PCR}

Total RNA was extracted from gastrocnemius muscle using Trizol reagent (Invitrogen, United States). The NanoDrop spectrophotometer and agarose gel electrophoresis were used to detect RNA concentration and integrity, respectively. Reverse transcription of mRNA into cDNA was carried out with PrimeScript RT master mix (Takara, Japan). And qPCR was performed using SYBR premix Ex Taq (Takara, Japan) with qTOWER 2.2 (Analytik Jena, Germany). The expression of target genes was normalized to $\beta$-actin. The primers sequences are listed in Supplementary Table 1.

\section{Determination of Mitochondrial DNA Copy Number}

mtDNA was extracted from gastrocnemius muscle using Mito DNA Extraction Kit (Genmed Scientifics). Cytochrome c oxidase II (COX2) was used for the quantification of the mtDNA copy number, whereas $18 \mathrm{~S}$ nuclear gene were used for standardization. The primers sequences are listed in Supplementary Table 1. 


\section{Statistical Analysis}

Values are expressed as the mead \pm SD and statistical analyses were performed using SPSS statistics 19.0 unless otherwise specified. Statistical analyses were applied using the unpaired 2 -tailed Student's $t$-test (for two groups) and one-way analysis of variance followed by LSD post hoc tests (for multiple groups comparisons). Correlation analysis was carried out using the Pearson with $\mathrm{R}$ software. In all cases, $p<0.05$ was considered statistically significant. And asterisks denote statistical significance (ns, no significance; ${ }^{*} p<0.05 ;{ }^{* *} p<0.01$; $\left.{ }^{* * *} p<0.001\right)$.

\section{RESULTS}

\section{Hypoxia Exposure Improved Endurance Performance of Mice}

To assess the effects of hypoxia on physical performance, mice were exposed to the normoxia environment or the hypoxic environment with $16.4 \%$ oxygen content for 4 weeks. As expected, the levels of $\mathrm{HGB}$ and $\mathrm{RBC}$ of hypoxia group were significantly increased in mice (Figures $\mathbf{1 A}, \mathbf{B}$ ). As shown in Figure 1C, hypoxia exposure significantly reduced body weight of mice, although food intake has no significant difference (Supplementary Figure 2A). Next, we investigated the effects of hypoxia on endurance performance. As shown in Figure 1D, the average time to exhaustion in hypoxia group increased compared with that in normoxia mice. Moreover, hypoxia treatment significantly decreased serum CK, BUN, and blood lactic acid levels, which were used to evaluate the degree of muscle fatigue and muscle damage (Figures 1E-G). In addition, blood glucose of hypoxia group was significantly increased ( $p=0.0064)$ compared with normoxia group, and there was no significant difference in hepatic glycogen between the two groups (Figures 1H,I). The RT-PCR analysis indicated that the mRNA expressions of PGC-1 $\alpha$, Tfam, and VEGF of gastrocnemius in hypoxia-exposed mice were significantly increased (Figure 1J). This finding was in line with the quantitative analysis of mtDNA by RT-PCR (Figure 1K) and TEM images (Figures $\mathbf{1 L}, \mathbf{M}$ ). However, we observed that hypoxia exposure did not change grip strength but decreased soleus and gastrocnemius muscles mass (Supplementary Figures 2B-D). Taken together, these results suggested that hypoxia exposure remarkably improved endurance performance but not muscle strength, and promoted mitochondrial biogenesis in skeletal muscle of mice.

\section{Hypoxia Treatment Modified the Gut Microbiota}

To investigate the effect of hypoxia exposure on the gut microbiota, fecal samples were harvested at the end of experiment, and the V3 and V4 $16 \mathrm{~S}$ rRNA variable gene regions were amplified and sequenced using the Illumina MiSeq platform. Our result showed no significant effects on $\alpha$-diversity between the two groups (Supplementary Figures 3A-D). Moreover, a clear separation based on the unweighted UniFrac distances was observed, suggesting that the gut microbiota was substantially remodeled by hypoxia exposure (Figure 2A). Furthermore, at the phylum level, hypoxia treatment markedly increased the abundance of Verrucomicrobia (Figures 2B,C), which was related to exercisedriven changes (Kim and Kang, 2019; Zhong et al., 2021). On the family level, we found that hypoxia increased the abundance of the Lactobacillaceae, Prevotellaceae, Akkermansiaceae, and Bacteroidaceae (Figures 2D,E). Further analysis at the genus level indicated an increase in the abundance of Lactobacillus, Prevotellaceae_UCG-001, Akkermansia, and Bacteroides, whereas reduced nclassified_f__Lachnospiraceae and norank_f_Lachnospiraceae (Figures $2 \mathbf{F}, \mathbf{G}$ ). The results of the LEfSe indicated that hypoxia caused an increase in the relative abundance of members from the phylum Verrucomicrobia, class Verrucomicrobiae, family Prevotellaceae, and genus Akkermansia relative to their levels in the normoxia mice (Figure 2H). Furthermore, the relative abundance of the family Peptococcaceae and genus Blautia were lower in the hypoxia group (Figure $2 \mathrm{H}$ ). Further correlation analysis showed that the fecal Lactobacillus, Akkermansia, and Bacteroides abundance were significantly negatively correlated with CK, BUN, and lactic acid levels (Figure 2I and Supplementary Figures 3E-G). The result was in line with some previous studies, which indicated that athletes had a higher abundance of indicated microbiota (Petersen et al., 2017; Huang et al., 2020). Taken together, these results indicated that hypoxia exposure modified gut microbiota composition.

\section{Microbial Changes Induced by Hypoxia Exposure Regulated Short-Chain Fatty Acids Production}

As accumulating evidence indicated that SCFAs contributed to exercise endurance (Okamoto et al., 2019; Scheiman et al., 2019; Bongiovanni et al., 2021), we measured the content of SCFAs in cecal contents to determine whether the hypoxiainduced enhancement of endurance capacity could be linked to the changes in SCFAs production. The results showed that hypoxia exposure significantly increased the contents of acetate, propionate, and butyrate (Figures 3A-C). Further correlation analysis showed that the contents of SCFAs were significantly positively correlated with exhaustion time, and negatively correlated with CK, BUN, and lactic acid levels (Figures 3DF). Interestingly, we found Akkermansia and Bacteroides were the key microbiota to produce SCFAs (Figure 3G) as reported (Koh et al., 2016). These results indicated that microbial changes induced by hypoxia exposure significantly increased the contents of SCFAs.

\section{Gut Microbiota Contributed to the Beneficial Effects of Hypoxia on Endurance Performance}

To determine whether the gut microbiota contributes to the beneficial effects of hypoxia on endurance performance, the experiment including antibiotic treatment and FMT was performed. The results showed that the beneficial effects of hypoxia on endurance performance were inhibited by $\mathrm{Abx}$ 


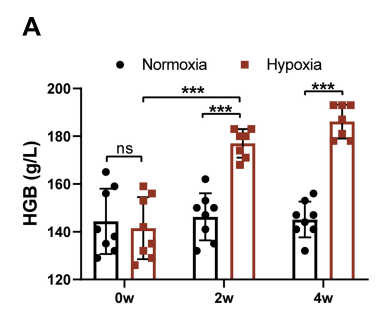

B

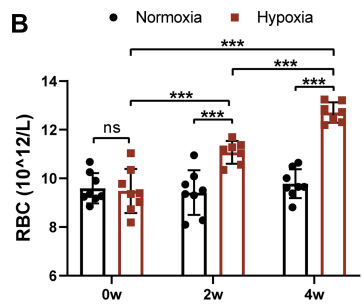

E

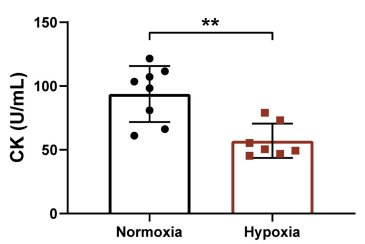

F

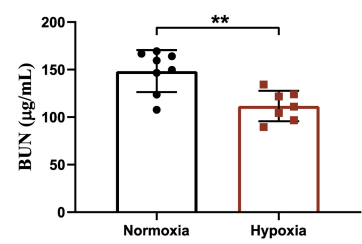

J

I
C

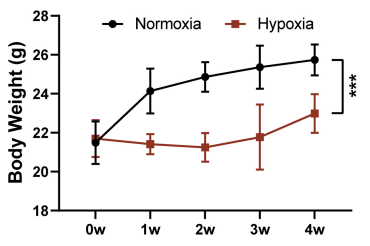

G

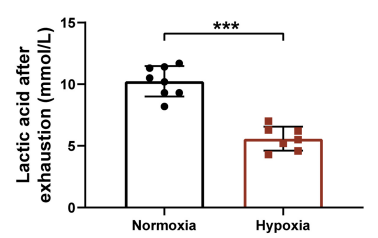

D

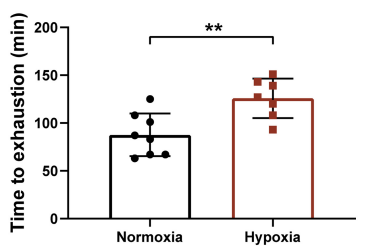

H

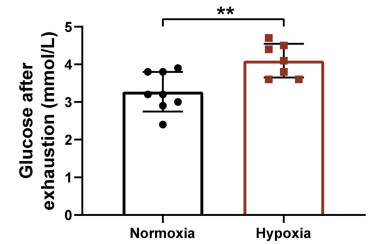

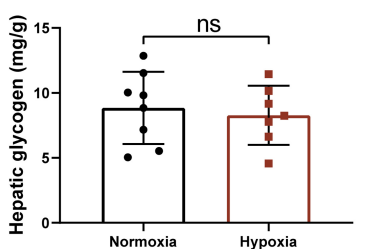

x

K

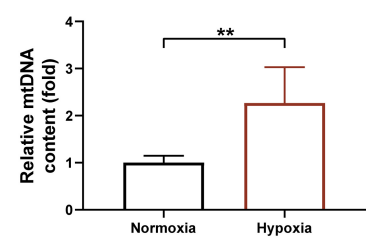

L

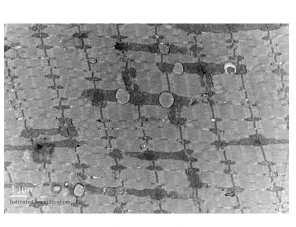

Normoxia
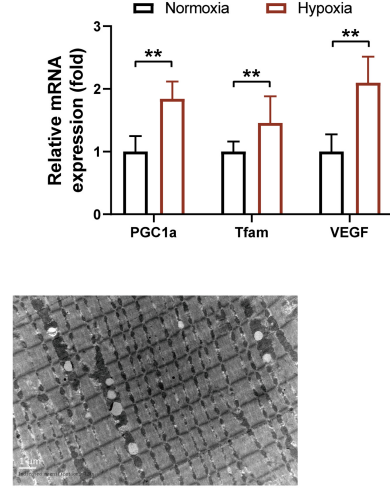

M

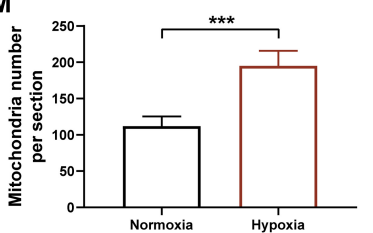

FIGURE 1 | Hypoxia exposure improved endurance performance of mice. HGB (A) and RBC (B) in the tail blood were detected. (C) Body weight was measured once a week. (D) Time to exhaustion was recorded. After exhaustion, CK (E), BUN (F), lactic acid (G), and glucose (H) were measured immediately. (I) The hepatic glycogen was measured. The expression of PGC- $1 \alpha$, Tfam, VEGF (J), and the mtDNA (K) content were measured by RT-PCR. (L) Representative TEM images of gastrocnemius at a magnification of 12,000x. (M) Quantification of the number of mitochondria per section. Data are presented as means \pm SD. ${ }^{*} p<0.05$, ${ }^{\star \star} p<0.01,{ }^{\star \star *} p<0.001$ (Student's $t$-test).

treatment (Figures 4A-D). Furthermore, we observed that Abx treatment decreased mitochondrial biogenesis (Figures $4 \mathrm{E}-\mathrm{H}$ ). However, Abx treatment had no influence on hematological parameters (Supplementary Figures 4A-C). In addition, Abx treatment reduced the gastrocnemius muscle weight and grip strength (Supplementary Figures 4D-F), which was in line with reported before (Lahiri et al., 2019).

To investigate whether the hypoxia-induced gut microbiota shift contributes to the increased endurance capacity and SCFAs concentration, the fecal suspensions derived from the normoxia and hypoxia-treated mice were transplanted into microbiotadepleted mice. We found that the effects of hypoxia on SCFAs concentration were transferable by the gut microbiota (Supplementary Figures 5A-C). Furthermore, transplantation of gut microbiota from the hypoxia-treated mice increased the endurance performance of receipt mice (Figures 5A-D). Moreover, this finding was in line with the increased skeletal muscle mitochondrial biogenesis observed in the receipt mice (Figures 5E-H). However, gut microbiota transplantation had no influence on hematological parameters, muscle weights, and grip strength (Supplementary Figures 5D-I). These results collectively suggested that the beneficial effects of hypoxia on endurance performance and mitochondrial biogenesis were partly dependent on the gut microbiota.

\section{Short-Chain Fatty Acids Supplementation Enhanced Endurance Capacity and Promoted Mitochondrial Biogenesis}

Previous studies reported that SCFAs mediate metabolic crosstalk between the gut microbiota and skeletal muscle (Okamoto et al., 2019; Frampton et al., 2020). SCFAs were also found to enhance mitochondrial biogenesis and mitochondrial function (Oliveira et al., 2015; Pirozzi et al., 2020). Thus, we hypothesized that SCFAs treatment might strengthen endurance capacity by increasing mitochondrial biogenesis. To test this hypothesis, mice were treated with vehicle, acetate, propionate, or butyrate 


\section{A}

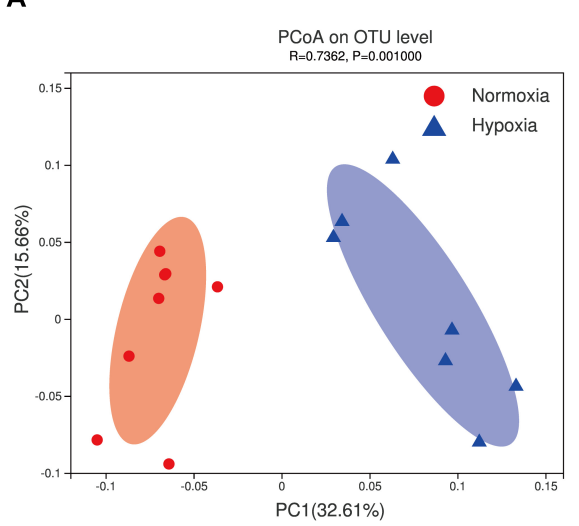

D

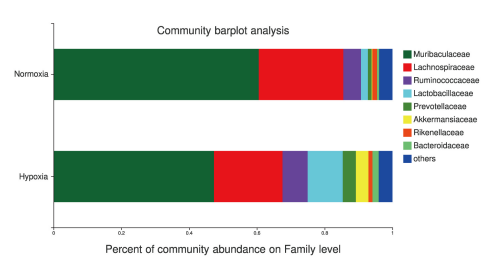

E
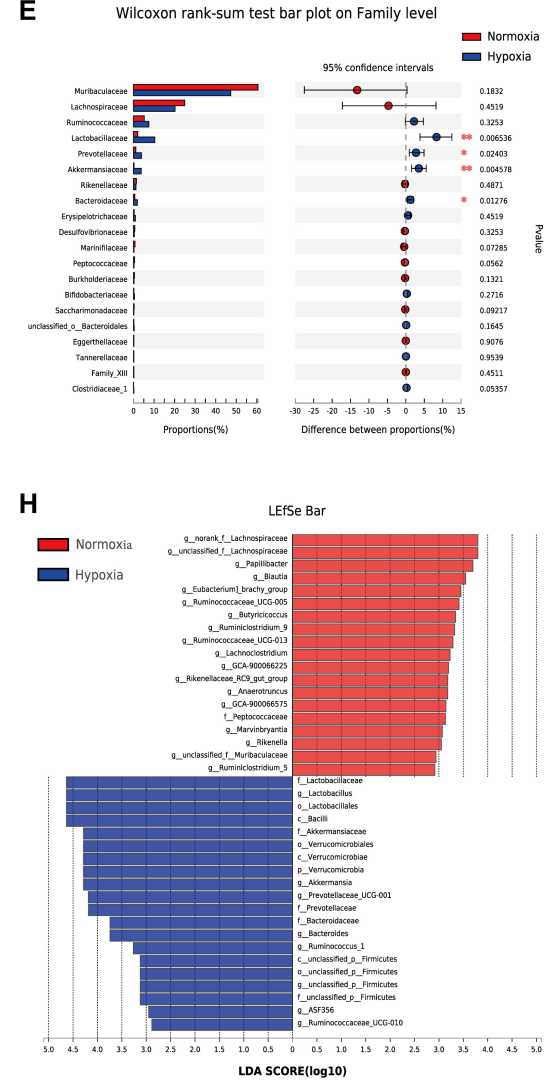

B

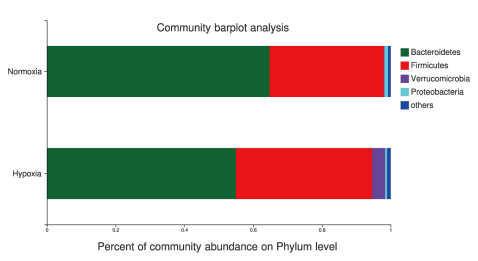

C

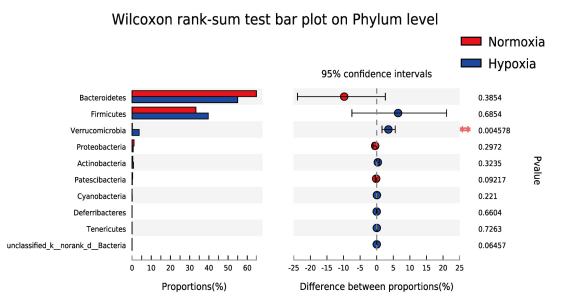

$\mathbf{F}$

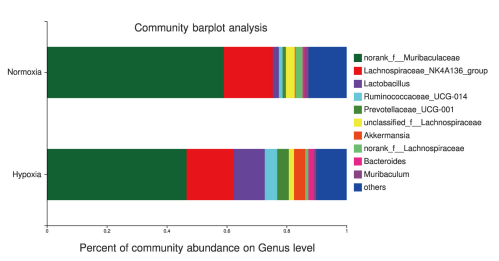

G

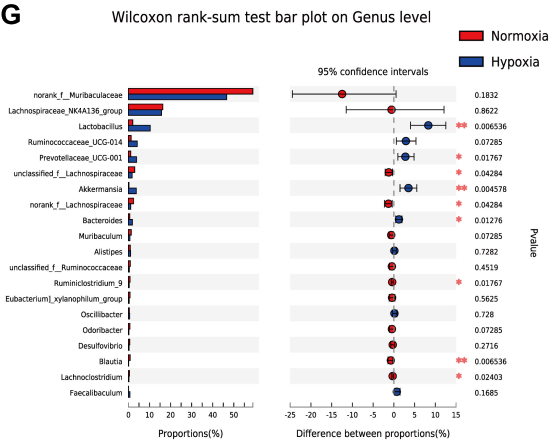

I

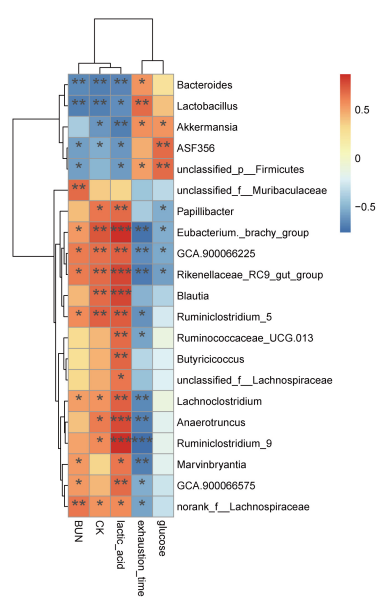

FIGURE 2 | Hypoxia treatment modified the gut microbiota. (A) Principal coordinate analysis (PCoA) of the $\beta$-diversity based on the unweighted UniFrac distance matrix (at the OTU level). Taxonomic distributions at the phylum level (B), and the Kruskal-Wallis $H$ test bar plot at the phylum level (C). Taxonomic distributions at the family level (D), and the Kruskal-Wallis $H$ test bar plot at the family level (E). Taxonomic distributions at the genus level (F), and the Kruskal-Wallis $H$ test bar plot at the genus level (G). (H) Bar graph of linear discriminant analysis (LDA) scores, showing the biomarker taxa (LDA score of $>2$ and a significance of $p<0.05$ determined by the Wilcoxon signed-rank test). (I) Heatmap of Pearson's correlation analysis between the key gut microbial taxa at the genus level and fatigue related factors. ${ }^{*} p<0.05,{ }^{* *} p<0.01,{ }^{* \star *} p<0.001$. 

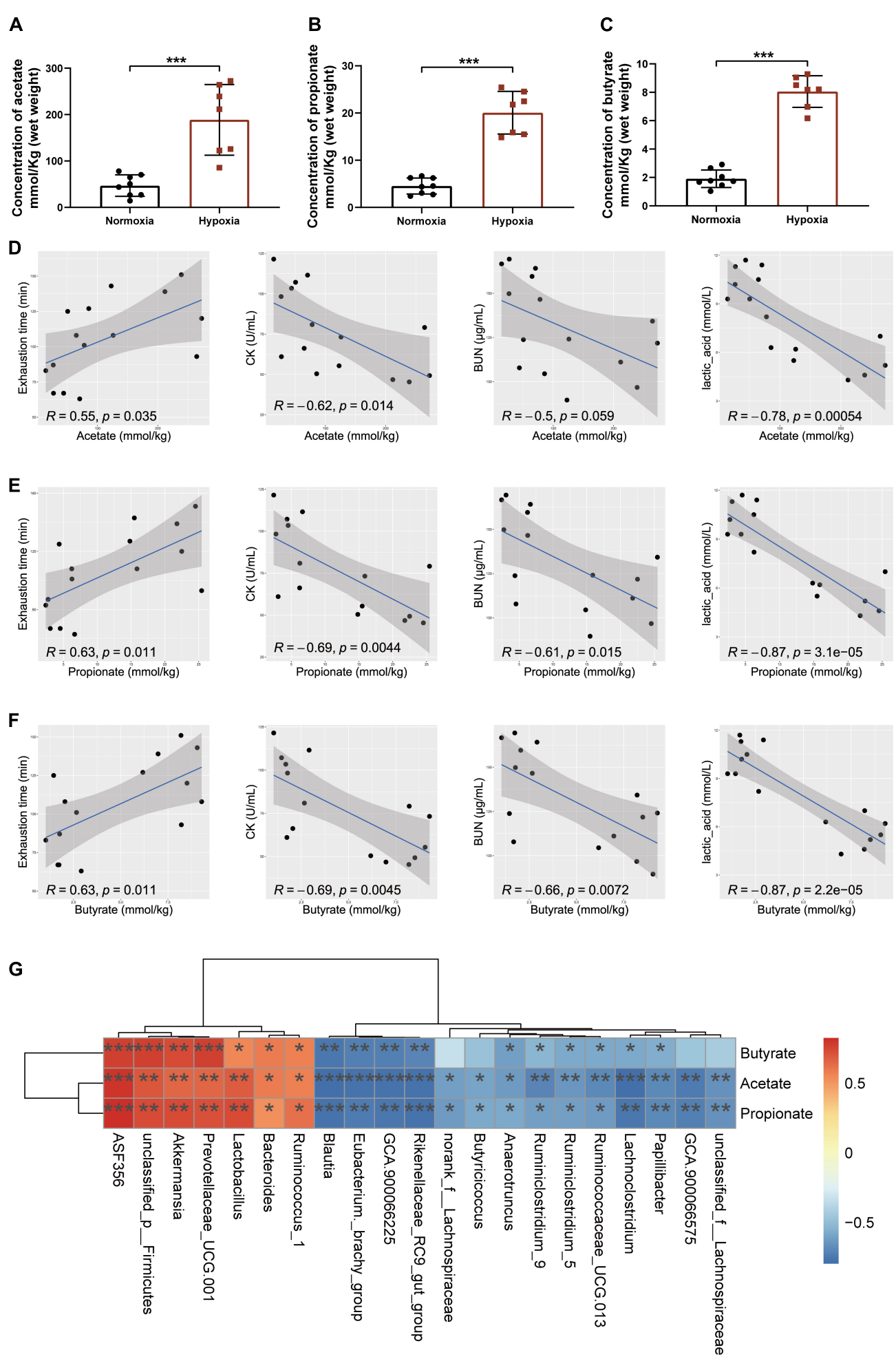

FIGURE 3 | Microbial changes induced by hypoxia exposure regulated SCFAs production. The contents of acetate (A), propionate (B), and butyrate (C) were measured by GC-MS in cecal contents. Data are presented as means \pm SD. (D) Plots show the relationship between the content of acetate and exhaustion time, CK, BUN, and lactic acid, represented as a smoothing spline with a 95\% confidence interval (shaded region). (E) Plots show the relationship between the content of propionate and exhaustion time, CK, BUN, and lactic acid, represented as a smoothing spline with a 95\% confidence interval (shaded region). (F) Plots show the relationship between the content of butyrate and exhaustion time, CK, BUN, and lactic acid, represented as a smoothing spline with a $95 \%$ confidence interval (shaded region). (G) Heatmap of Pearson's correlation analysis between the key gut microbial taxa at the genus level and the contents of SCFAs in cecal contents. ${ }^{*} p<0.05,{ }^{* *} p<0.01,{ }^{* \star *} p<0.001$. (A-C, Student's $t$-test; $\mathbf{D}-\mathbf{G}$, Pearson's correlation). 
A

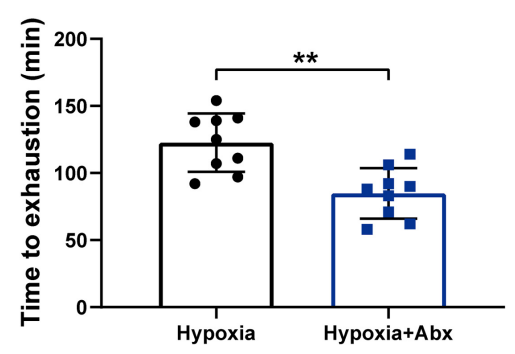

D

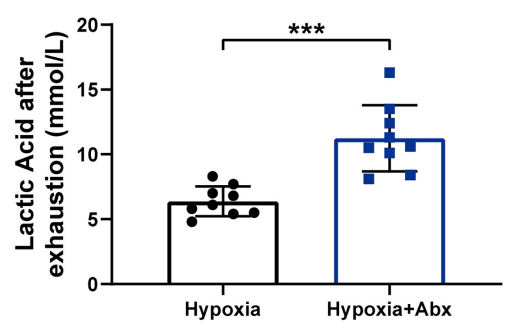

G

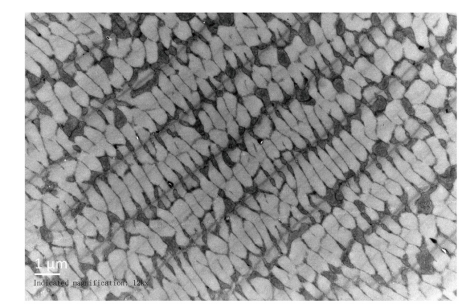

Hypoxia
B

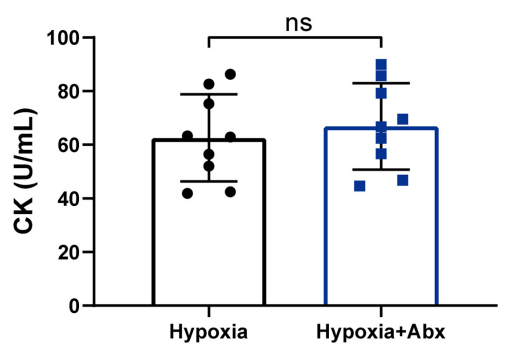

C

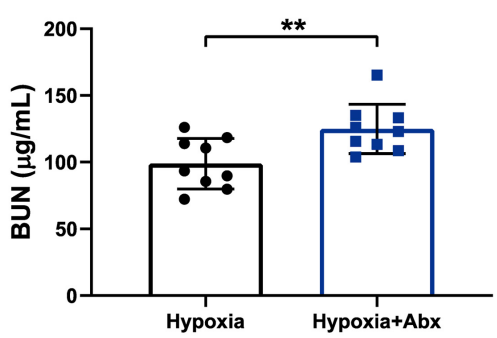

$E$

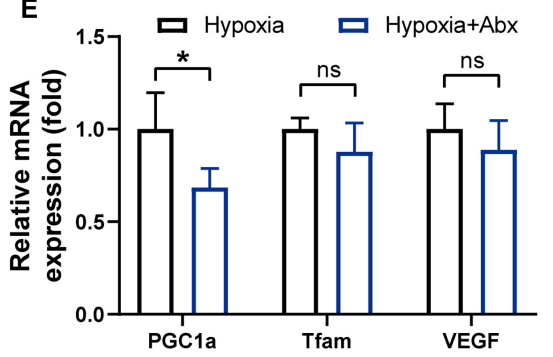

$\mathbf{F}$

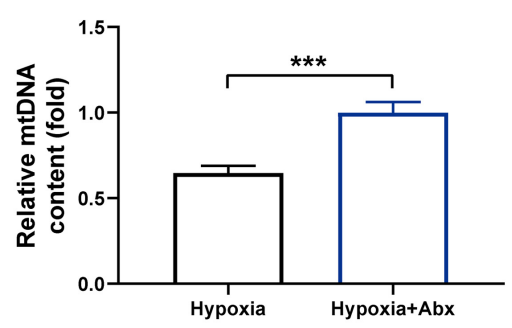

H

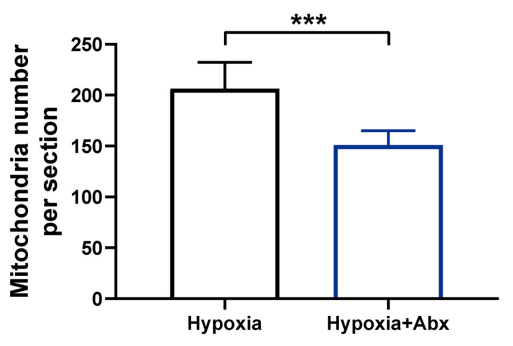

FIGURE 4 | Gut microbiota contributed to the beneficial effects of hypoxia on endurance performance. (A) Time until exhaustion was recorded. After exhaustion, CK (B), BUN (C), and lactic acid (D) were measured immediately. The expression of mitochondrial biogenesis (E) and the mtDNA content (F) were measured by RT-PCR. Representative TEM images (G) and quantification (H) of mitochondria in gastrocnemius muscle at original magnification of $\times 12,000$. Data are presented as means $\pm \mathrm{SD}$. ${ }^{\star} p<0.05,{ }^{* *} p<0.01,{ }^{* \star *} p<0.001$ (Student's $t$-test, ns, no significance).

for 4 weeks as indicated. Figure $\mathbf{6 A}$ showed SCFAs treatment did not affect body weight. Moreover, acetate and butyrate treatment significantly improved the average running time compared with the control group (Figure 6B). Furthermore, the levels of CK (Figure 6C) and BUN (Figure 6D) decreased in acetate and butyrate-treated groups, but there was no significant difference in lactic acid concentration between the four groups (Figure 6E). In addition, acetate and butyrate treatment significantly increased the mRNA expression levels of mitochondrial biogenesis-related genes in gastrocnemius compared with vehicle treatment (Figure 6F). The quantitative analysis of mitochondria DNA by RT-PCR (Figure 6G) and TEM (Figures 6H,I) revealed that the number of intermyofibrillar mitochondria in acetate and butyrate-treated groups significantly increased compared to the vehicle and propionate-treated groups. Taken together, these results suggested that acetate and butyrate could enhance endurance capacity by promoting mitochondrial biogenesis.

\section{DISCUSSION}

Although hypoxia exposure has been widely used to improve athletic endurance performance, the underlying mechanisms have not been fully clarified. Our results suggested that hypoxia markedly improved endurance capacity by promoting hematological parameters and muscular mitochondrial biogenesis in mice. We further demonstrated that normobaric hypoxia exposure remodeled the gut microbiota, leading to an increase in SCFAs production, which were highly correlated with endurance capacity. In addition, removing gut microbiota by antibiotic treatment abolished the effects of hypoxia on endurance capacity and muscular mitochondrial biogenesis. Moreover, the hypoxia-induced increase in the production of fecal SCFAs and enhancement of endurance capacity could be transferred to the microbiota-depleted mice by FMT. These results indicated that the gut microbiota played an essential role in the hypoxia-induced endurance capacity enhancement. 
A

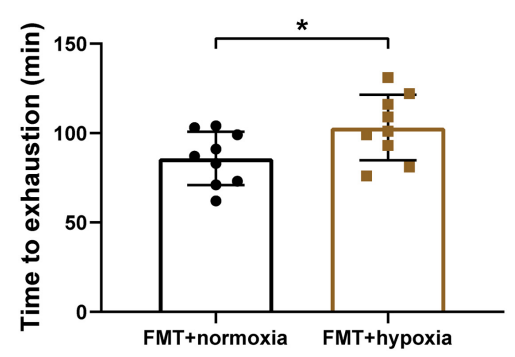

D

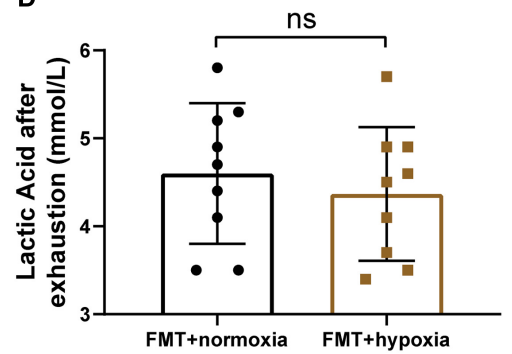

G

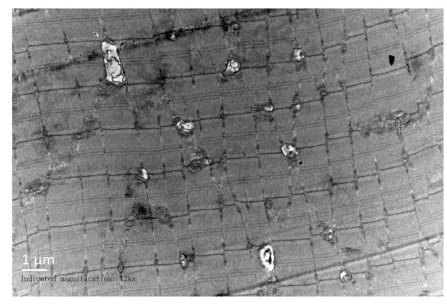

FMT+normoxia
B

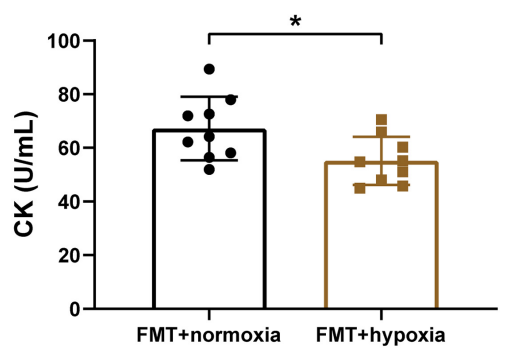

E
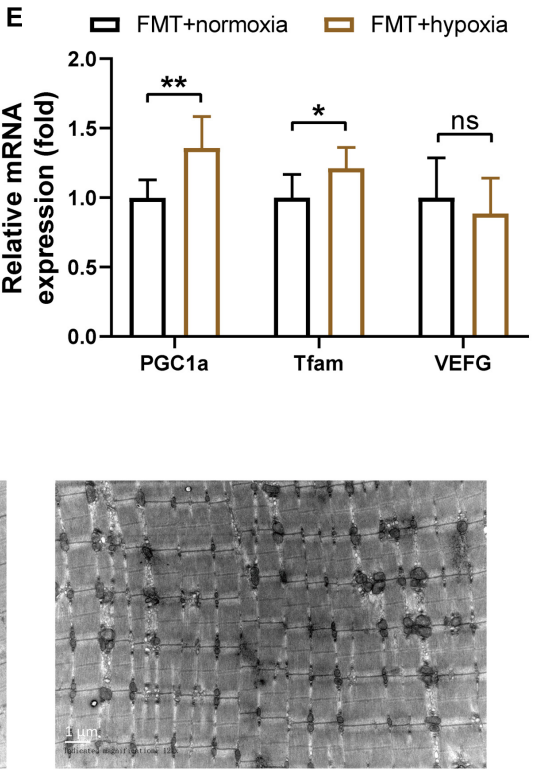

FMT+hypoxia
F

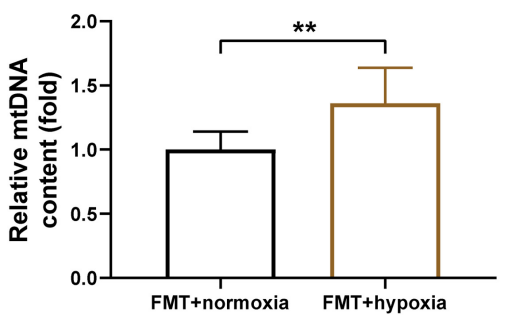

H

C
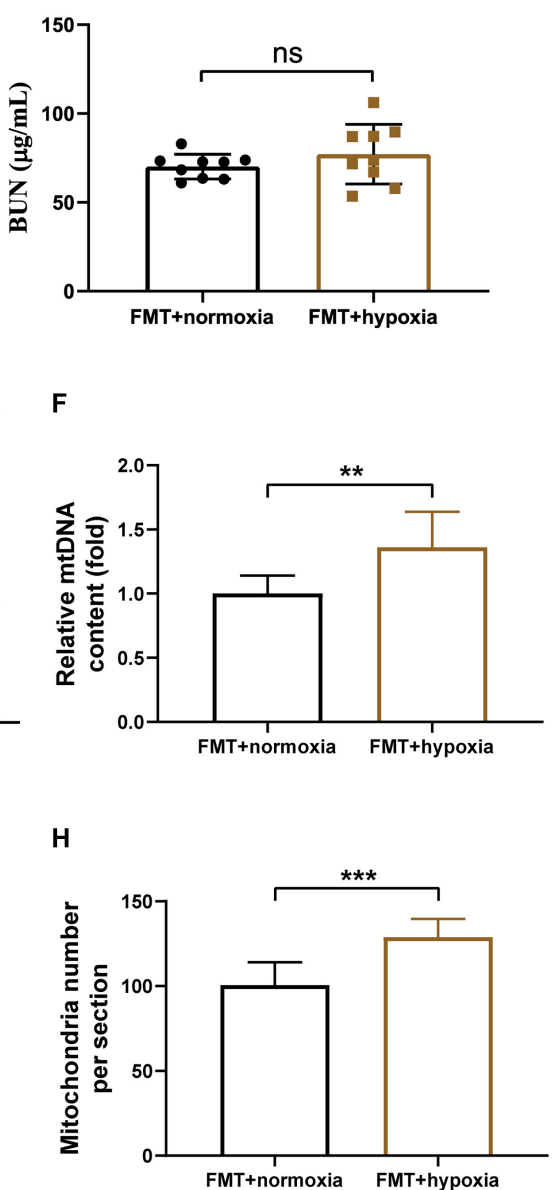

FIGURE 5 | SCFAs supplementation enhanced endurance capacity and promoted mitochondrial biogenesis. (A) Running time until exhaustion was recorded. CK (B), BUN (C), and lactic acid (D) were measured immediately after exhaustion. The expression of mitochondrial biogenesis (E) and the mtDNA content (F) were measured by RT-PCR. (G) Representative TEM images of gastrocnemius at a magnification of 12,000x. (H) Quantification of the number of mitochondria per section. Data are presented as means \pm SD. ${ }^{*} p<0.05,{ }^{* *} p<0.01,{ }^{\star \star *} p<0.001$ (Student's $t$-test, ns, no significance).

Furthermore, we demonstrated that acetate and butyrate supplementation significantly increased muscular mitochondrial biogenesis, thus enhancing endurance capacity in mice. Taken together, these results demonstrated that the beneficial effects of hypoxia exposure on endurance performance were partially achieved via remodeling of the gut microbiota, and consequently increasing the production of intestinal SCFAs.

Previous studies have reported that hypoxia could significantly affect the gut microbiota (Bai et al., 2018; Mazel, 2019; Han et al., 2021), but the understanding of hypoxia-induced changes in the gut microbiota is far from clear. It was widely accepted that hypoxia exposure significantly decreased the aerobic bacteria and increased the anaerobic bacteria (Mazel, 2019; Han et al., 2021). However, most studies have not excluded the interference of confounding factors such as genes, diet, exercise, and hypoxia exposure model (Li and Zhao, 2015; Karl et al., 2018; Suzuki et al., 2019; Jia et al., 2020; Montoya-Ciriaco et al., 2020). Kleessen et al. (2005) reported that mountaineers exposed to high altitudes above 5,000 $\mathrm{m}$ have decreased beneficial Bifidobacteria and increased potentially pathogenic gram-negative bacteria such as Enterobacteriaceae that bring health risks. Moreover, strict anaerobes such as Lactobacillus and Bacteroidetes and obligate anaerobes such as Clostridium perfringens and Escherichia coli were increased in soldiers exposed to high altitudes at $3,505 \mathrm{~m}$ for 15 days (Adak et al., 2013). These results indicated that different levels of atmospheric oxygen might have different effects on the gut microbiota. However, little research focused on the effects of moderate altitude (2,000-3,000 m) on the gut microbiota. In addition, different from short-term exposure to severe hypoxia environment, plateau-living humans or animals showed a rich intestinal microbiota which helps the hosts to adapt to the plateau environment (Li et al., 2016, 2018, 2019; Jia et al., 2020; Zeng et al., 2021). All these studies showed the complex and varied effects of hypoxia on the gut microbiota. We conducted a randomized controlled animal study, which excluded confounding factors (including temperature, nutrition, atmospheric pressure, etc.). 


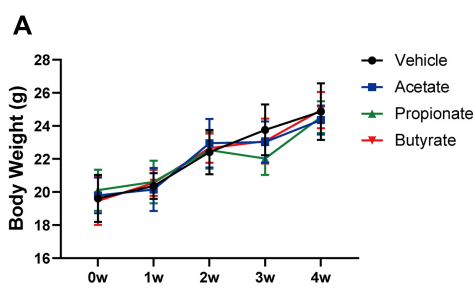

E

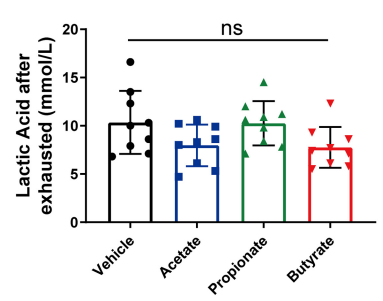

H

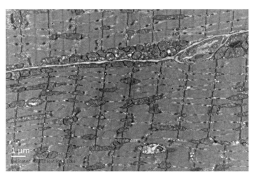

Vehicle
B

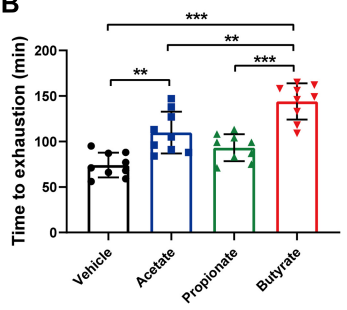

$\mathbf{F}$

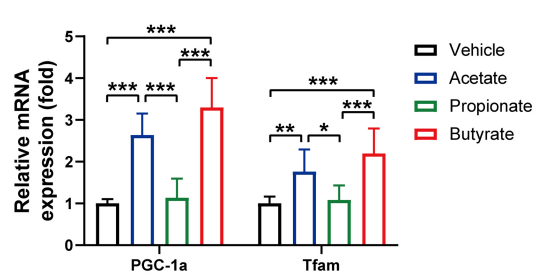

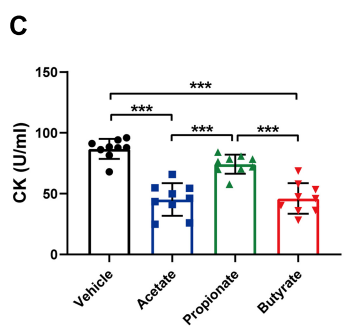

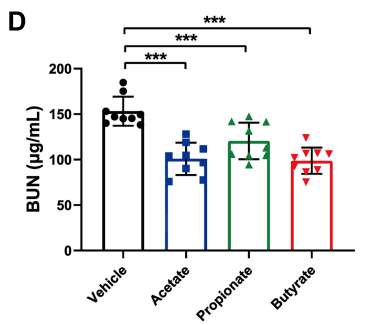

G
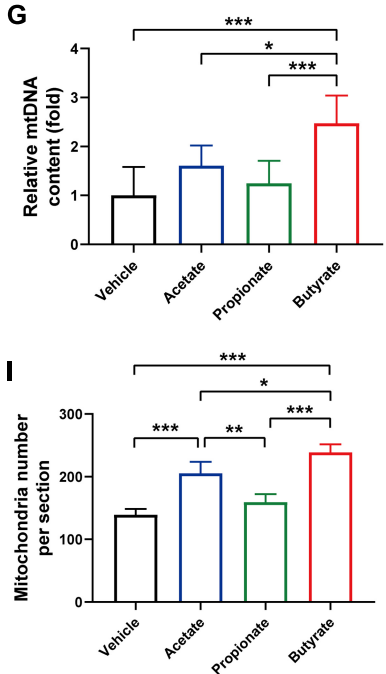

FIGURE 6 | SCFAs supplement enhanced endurance capacity by promoting mitochondrial synthesis. (A) Body weight was measured once a week. (B) Time to exhaustion was recorded. After exhaustion, CK (C), BUN (D), and lactic acid (E) were measured immediately. RT-PCR analysis of mitochondrial biogenesis gene expression (F) and mtDNA content (G) in gastrocnemius. Representative TEM images $\mathbf{( H )}$ and quantification (I) of mitochondria in gastrocnemius muscle at original magnification of $\times 12,000$. Data are presented as means \pm SD. ${ }^{*} p<0.05,{ }^{\star *} p<0.01,{ }^{\star \star *} p<0.001$ (One way ANOVA).

Our results indicated that exposure to the normobaric normoxia environment $\left(16.4 \% \mathrm{O}_{2}\right.$, equivalent to an altitude of $2,000 \mathrm{~m}$ ) for 4 weeks significantly increased the abundance of Lactobacillus, Prevotellaceae_UCG-001, Akkermansia, and Bacteroides at the genus level, accompanied by an increase of fecal acetate, propionate, and butyrate production in mice.

Moderate altitude training has emerged as a popular method to improve athletic endurance performance (Girard et al., 2013a; Millet and Brocherie, 2020). However, the underlying mechanisms have not been fully clarified (Girard et al., 2013a; Zhao et al., 2018; Millet and Brocherie, 2020; Siebenmann and Dempsey, 2020). Recent evidence indicated that exercise and the gut microbiota were interconnected (Przewłócka et al., 2020; Clauss et al., 2021). Several studies reported that endurance athletes had a higher abundance of Prevotella and Akkermansia, and a lower abundance of Bacteroidetes and Lactobacillus, accompanied by an accumulation of fecal SCFAs production (Clarke et al., 2014; Barton et al., 2018; Kulecka et al., 2020). Moreover, a single or multiple exercises could remodel the structure of the gut microbiota (Munukka et al., 2018; Scheiman et al., 2019; Kern et al., 2020). On the other hand, it was found that the metabolites of gut microbiota could participate in energy metabolism or act as an energy substrate to improve exercise performance. Frampton et al. (2020) indicated that Veillonella could metabolize lactate into acetate and propionate, thus improving endurance ability. In addition, Okamoto et al. (2019) suggested that acetate could act as a metabolic fuel for skeletal muscle. This study suggested that hypoxia improved endurance performance in mice by increasing the abundance of SCFAproducing bacteria, including Akkermansia and Bacteroidetes, thereby resulting in an increase of fecal SCFAs production. Moreover, the antibiotic treatment and FMT experiments suggested that the beneficial effects of hypoxia on endurance performance and SCAFs production were partly dependent on the gut microbiota. In summary, these results implied that gut microbiota might play a critical role in hypoxia enhancing endurance performance.

SCFAs, the metabolites derived from microbial fermentation of dietary fibers, have been demonstrated to regulate host energy balance, nutrition metabolism, and immune function (Koh et al., 2016; Makki et al., 2018). Accumulating evidence indicated that SCFAs participate in protein, carbohydrate, and lipid metabolism in skeletal muscle (Frampton et al., 2020). Moreover, SCFAs could enhance the athlete's immunity, improve exercise recovery via anti-inflammatory activity and provide additional energy substrates for exercise performance (Frampton et al., 2020; Bongiovanni et al., 2021). Furthermore, it has been demonstrated that the muscle oxidative capacity, which 
is strongly correlated with the muscle mitochondrial content, was a major determinant of endurance capacity (Irrcher et al., 2003; Booth et al., 2015). Additionally, the inhibition of histone deacetylases and the activation of AMPK, PPAR- $\delta$, and PGC$1 \alpha$ are likely key mechanisms through which SCFAs promote exercise performance (Frampton et al., 2020; Bongiovanni et al., 2021). In this study, we found that the contents of SCFAs were significantly positively correlated with endurance performance. Furthermore, our results indicated that that acetate and butyrate supplementation, but not propionate, promoted skeletal muscle mitochondrial biogenesis and enhanced endurance performance in mice. Taken together, our results suggested that the hypoxia-induced changes in SCFAs production contribute to the enhanced endurance capacity by promoting muscle mitochondrial biogenesis.

\section{CONCLUSION}

In summary, our results have enriched the understanding of hypoxia exposure reshaping the gut microbiota. Moreover, our study suggested that the beneficial effects of the hypoxia on endurance performance were partly dependent on the increased production of SCFAs resulting from the remodeling of the gut microbiota. Overall, our study indicated that hypoxia exposure improves exercise performance partially via regulation of the gut microbiota-SCFAs axis.

\section{DATA AVAILABILITY STATEMENT}

The raw data of $16 \mathrm{~S}$ rRNA sequencing was deposited in NCBI Sequence Read Archive (SRA) accession no. PRJNA778424. Available online at: https://www.ncbi.nlm.nih.gov/bioproject/ PRJNA778424.

\section{REFERENCES}

Adak, A., Maity, C., Ghosh, K., Pati, B. R., and Mondal, K. C. (2013). Dynamics of predominant microbiota in the human gastrointestinal tract and change in luminal enzymes and immunoglobulin profile during highaltitude adaptation. Folia Microbiol. 58, 523-528. doi: 10.1007/s12223-0130241-y

Bai, X., Lu, S., Yang, J., Jin, D., Pu, J., Díaz Moyá, S., et al. (2018). Precise fecal microbiome of the herbivorous tibetan antelope inhabiting high-altitude Alpine Plateau. Front. Microbiol. 9:2321. doi: 10.3389/fmicb.2018.02321

Barton, W., Penney, N. C., Cronin, O., Garcia-Perez, I., Molloy, M. G., Holmes, E., et al. (2018). The microbiome of professional athletes differs from that of more sedentary subjects in composition and particularly at the functional metabolic level. Gut 67, 625-633. doi: 10.1136/gutjnl-2016-313627

Bongiovanni, T., Yin, M. O. L., and Heaney, L. (2021). The athlete and gut microbiome: short-chain fatty acids as potential ergogenic aids for exercise and training. Int J Sports Med. 42, 1143-1158. doi: 10.1055/a-1524-2095

Booth, F. W., Ruegsegger, G. N., Toedebusch, R. G., and Yan, Z. (2015). Endurance exercise and the regulation of skeletal muscle metabolism. Prog. Mol. Biol. Transl. Sci. 135, 129-151. doi: 10.1016/bs.pmbts.2015.07.016

Caporaso, J. G., Kuczynski, J., Stombaugh, J., Bittinger, K., Bushman, F. D., Costello, E. K., et al. (2010). QIIME allows analysis of high-throughput community sequencing data. Nat. Methods 7, 335-336. doi: 10.1038/nmet h.f.303

\section{ETHICS STATEMENT}

The animal study was reviewed and approved by the Animal Care and Use Committee of the Third Military Medical University.

\section{AUTHOR CONTRIBUTIONS}

MM and XZ conceived and designed the study. LH, MZ, TL, and MD performed the experiments and collected the samples. $\mathrm{LH}$, LZ, LY, and MZ analyzed the data. LH, JZ, XZ, and MM wrote the manuscript. MM obtained funding. All authors contributed to the article and approved the submitted version.

\section{FUNDING}

This work was supported by the research grants from the Key Projects for Scientific Research (AWS17J014).

\section{ACKNOWLEDGMENTS}

We thank Changjun Zhao and Jing Yue for assistance with animal tissue collection.

\section{SUPPLEMENTARY MATERIAL}

The Supplementary Material for this article can be found online at: https://www.frontiersin.org/articles/10.3389/fmicb. 2021.820691/full\#supplementary-material

Clarke, S. F., Murphy, E. F., O’Sullivan, O., Lucey, A. J., Humphreys, M., Hogan, A. et al. (2014). Exercise and associated dietary extremes impact on gut microbial diversity. Gut 63, 1913-1920. doi: 10.1136/gutjnl-2013-306541

Clauss, M., Gérard, P., Mosca, A., and Leclerc, M. (2021). Interplay between exercise and gut microbiome in the context of human health and performance. Front. Nutr. 8:637010. doi: 10.3389/fnut.2021.637010

Edgar, R. C. (2013). UPARSE: highly accurate OTU sequences from microbial amplicon reads. Nat. Methods 10, 996-998. doi: 10.1038/nmeth.2604

Fachi, J. L., Felipe, J. S., Pral, L. P., da Silva, B. K., Corrêa, R. O., de Andrade, M. C. P., et al. (2019). Butyrate protects mice from clostridium difficile-induced colitis through an HIF-1-dependent mechanism. Cell Rep. 27, 750-761.e7. doi: 10.1016/j.celrep.2019.03.054

Fan, Y., and Pedersen, O. (2021). Gut microbiota in human metabolic health and disease. Nat. Rev. Microbiol. 19, 55-71. doi: 10.1038/s41579-020-04339

Frampton, J., Murphy, K. G., Frost, G., and Chambers, E. S. (2020). Short-chain fatty acids as potential regulators of skeletal muscle metabolism and function. Nat. Metab. 2, 840-848. doi: 10.1038/s42255-020-0188-7

Girard, O., Amann, M., Aughey, R., Billaut, F., Bishop, D. J., Bourdon, P., et al. (2013a). Position statement-altitude training for improving team-sport players' performance: current knowledge and unresolved issues. Br. J. Sports Med. 47(Suppl. 1), i8-i16. doi: 10.1136/bjsports-2013-093109

Girard, O., Brocherie, F., and Millet, G. P. (2013b). On the use of mobile inflatable hypoxic marquees for sport-specific altitude training in team sports. Br. J. Sports Med. 47(Suppl. 1), i121-i123. doi: 10.1136/bjsports-2013-092794 
Han, N., Pan, Z., Huang, Z., Chang, Y., Hou, F., Liu, G., et al. (2020). Effects of Myeloid Hif- $1 \beta$ deletion on the intestinal microbiota in mice under environmental hypoxia. Infect. Immun. 89:e00474-20. doi: 10.1128/iai.00474-20

Han, N., Pan, Z., Liu, G., Yang, R., and Yujing, B. (2021). Hypoxia: the "Invisible Pusher" of Gut Microbiota. Front. Microbiol. 12:690600. doi: 10.3389/fmicb. 2021.690600

Hobbins, L., Hunter, S., Gaoua, N., and Girard, O. (2017). Normobaric hypoxic conditioning to maximize weight loss and ameliorate cardio-metabolic health in obese populations: a systematic review. Am. J. Physiol. Regul. Integr. Comp. Physiol. 313, R251-R264. doi: 10.1152/ajpregu.00160.2017

Hou, P., Zhou, X., Yu, L., Yao, Y., Zhang, Y., Huang, Y., et al. (2020). Exhaustive exercise induces gastrointestinal syndrome through reduced ILC3 and IL-22 in Mouse Model. Med. Sci. Sports Exerc. 52, 1710-1718. doi: 10.1249/mss. 0000000000002298

Hsu, Y. J., Chiu, C. C., Li, Y. P., Huang, W. C., Huang, Y. T., Huang, C. C., et al. (2015). Effect of intestinal microbiota on exercise performance in mice. J. Strength Cond. Res. 29, 552-558. doi: 10.1519/jsc.00000000000 00644

Hu, S., Kuwabara, R., de Haan, B. J., Smink, A. M., and de Vos, P. (2020). Acetate and Butyrate Improve $\beta$-cell metabolism and mitochondrial respiration under oxidative stress. Int. J. Mol. Sci. 21:1542. doi: 10.3390/ijms21041542

Huang, W. C., Chen, Y. H., Chuang, H. L., Chiu, C. C., and Huang, C. C. (2019). Investigation of the effects of microbiota on exercise physiological adaption, performance, and energy utilization using a Gnotobiotic Animal Model. Front. Microbiol. 10:1906. doi: 10.3389/fmicb.2019.01906

Huang, W. C., Pan, C. H., Wei, C. C., and Huang, H. Y. (2020). Lactobacillus plantarum PS128 improves physiological adaptation and performance in triathletes through Gut Microbiota Modulation. Nutrients 12:2315. doi: 10. 3390/nu12082315

Hui, S., Liu, Y., Huang, L., Zheng, L., Zhou, M., Lang, H., et al. (2020). Resveratrol enhances brown adipose tissue activity and white adipose tissue browning in part by regulating bile acid metabolism via gut microbiota remodeling. Int. J. Obes. 44, 1678-1690. doi: 10.1038/s41366-020-0566-y

Irrcher, I., Adhihetty, P. J., Joseph, A. M., Ljubicic, V., and Hood, D. A. (2003). Regulation of mitochondrial biogenesis in muscle by endurance exercise. Sports Med. 33, 783-793. doi: 10.2165/00007256-200333110

Jia, Z., Zhao, X., Liu, X., Zhao, L., Jia, Q., Shi, J., et al. (2020). Impacts of the Plateau Environment on the Gut Microbiota and Blood Clinical Indexes in Han and Tibetan Individuals. mSystems 5:e00660-19. doi: 10.1128/mSystems.00660-19

Kang, C., Wang, B., Kaliannan, K., Wang, X., Lang, H., Hui, S., et al. (2017). Gut Microbiota mediates the protective effects of dietary capsaicin against chronic low-grade inflammation and associated obesity induced by high-fat diet. mBio 8:e00470-17. doi: 10.1128/mBio.00470-17

Karl, J. P., Berryman, C. E., Young, A. J., Radcliffe, P. N., Branck, T. A., PantojaFeliciano, I. G., et al. (2018). Associations between the gut microbiota and host responses to high altitude. Am. J. Physiol. Gastrointest. Liver Physiol. 315, G1003-G1015. doi: 10.1152/ajpgi.00253.2018

Kayser, B., and Verges, S. (2013). Hypoxia, energy balance and obesity: from pathophysiological mechanisms to new treatment strategies. Obes. Rev. 14, 579-592. doi: 10.1111/obr.12034

Kern, T., Blond, M. B., Hansen, T. H., Rosenkilde, M., Quist, J. S., Gram, A. S., et al. (2020). Structured exercise alters the gut microbiota in humans with overweight and obesity-A randomized controlled trial. Int. J. Obes. 44, 125-135. doi: 10.1038/s41366-019-0440-y

Kim, D., and Kang, H. (2019). Exercise training modifies gut microbiota with attenuated host responses to sepsis in wild-type mice. FASEB J. 33, 5772-5781. doi: 10.1096/fj.201802481R

Kleessen, B., Schroedl, W., Stueck, M., Richter, A., Rieck, O., and Krueger, M. (2005). Microbial and immunological responses relative to high-altitude exposure in mountaineers. Med. Sci. Sports Exerc. 37, 1313-1318. doi: 10.1249/ 01.mss.0000174888.22930.e0

Koh, A., De Vadder, F., Kovatcheva-Datchary, P., and Bäckhed, F. (2016). From dietary fiber to host physiology: short-chain fatty acids as key bacterial metabolites. Cell 165, 1332-1345. doi: 10.1016/j.cell.2016.05.041

Kulecka, M., Fraczek, B., Mikula, M., Zeber-Lubecka, N., Karczmarski, J., Paziewska, A., et al. (2020). The composition and richness of the gut microbiota differentiate the top Polish endurance athletes from sedentary controls. Gut Microbes 11, 1374-1384. doi: 10.1080/19490976.2020.1758009
Lahiri, S., Kim, H., Garcia-Perez, I., Reza, M. M., Martin, K. A., Kundu, P., et al. (2019). The gut microbiota influences skeletal muscle mass and function in mice. Sci. Transl. Med. 11:eaan5662. doi: 10.1126/scitranslmed.a an5662

Li, H., Qu, J., Li, T., Wirth, S., Zhang, Y., Zhao, X., et al. (2018). Diet simplification selects for high gut microbial diversity and strong fermenting ability in high-altitude pikas. Appl. Microbiol. Biotechnol. 102, 6739-6751. doi: 10.1007/ s00253-018-9097-z

Li, H., Zhou, R., Zhu, J., Huang, X., and Qu, J. (2019). Environmental filtering increases with elevation for the assembly of gut microbiota in wild pikas. Microb. Biotechnol. 12, 976-992. doi: 10.1111/1751-7915.13450

Li, K., Dan, Z., Gesang, L., Wang, H., Zhou, Y., Du, Y., et al. (2016). Comparative Analysis of Gut Microbiota of Native Tibetan and Han Populations Living at Different Altitudes. PLoS One 11:e0155863. doi: 10.1371/journal.pone.0155863

Li, L., and Zhao, X. (2015). Comparative analyses of fecal microbiota in Tibetan and Chinese Han living at low or high altitude by barcoded 454 pyrosequencing. Sci. Rep. 5:14682. doi: 10.1038/srep14682

Liu, Y., Lang, H., Zhou, M., Huang, L., Hui, S., Wang, X., et al. (2020). The preventive effects of pterostilbene on the exercise intolerance and circadian misalignment of mice subjected to sleep restriction. Mol. Nutr. Food Res. 64, e1900991. doi: 10.1002/mnfr.201900991

Lundberg, J. O., Moretti, C., Benjamin, N., and Weitzberg, E. (2021). Symbiotic bacteria enhance exercise performance. Br. J. Sports Med. 55:243. doi: 10.1136/ bjsports-2020-102094

Ma, Y., Ga, Q., Ge, R. L., and Ma, S. (2021). Correlations between intestinal microbial community and hematological profile in native Tibetans and Han immigrants. Front. Microbiol. 12:615416. doi: 10.3389/fmicb.2021.615416

Mailing, L. J., Allen, J. M., Buford, T. W., Fields, C. J., and Woods, J. A. (2019). Exercise and the Gut Microbiome: a review of the evidence, potential mechanisms, and implications for human health. Exerc. Sport Sci. Rev. 47, 75-85. doi: 10.1249/jes.0000000000000183

Makki, K., Deehan, E. C., Walter, J., and Bäckhed, F. (2018). The impact of dietary fiber on gut microbiota in host health and disease. Cell Host Microbe 23, 705-715. doi: 10.1016/j.chom.2018.05.012

Mazel, F. (2019). Living the high life: Could gut microbiota matter for adaptation to high altitude? Mol. Ecol. 28, 2119-2121. doi: 10.1111/mec.15093

Millet, G. P., and Brocherie, F. (2020). Hypoxic training is beneficial in elite athletes. Med. Sci. Sports Exerc. 52, 515-518. doi: 10.1249/mss. 0000000000002142

Montoya-Ciriaco, N., Gómez-Acata, S., Muñoz-Arenas, L. C., Dendooven, L., Estrada-Torres, A., Díaz de la Vega-Pérez, A. H., et al. (2020). Dietary effects on gut microbiota of the mesquite lizard Sceloporus grammicus (Wiegmann, 1828) across different altitudes. Microbiome 8:6. doi: 10.1186/s40168-020-0783-6

Mujika, I., Sharma, A. P., and Stellingwerff, T. (2019). Contemporary periodization of altitude training for elite endurance athletes: a narrative review. Sports Med. 49, 1651-1669. doi: 10.1007/s40279-019-01165-y

Munukka, E., Ahtiainen, J. P., Puigbo, P., Jalkanen, S., Pahkala, K., Keskitalo, A., et al. (2018). Six-week endurance exercise alters gut metagenome that is not reflected in systemic metabolism in over-weight women. Front. Microbiol. 9:2323. doi: 10.3389/fmicb.2018.02323

Nay, K., Jollet, M., Goustard, B., Baati, N., Vernus, B., Pontones, M., et al. (2019). Gut bacteria are critical for optimal muscle function: a potential link with glucose homeostasis. Am. J. Physiol. Endocrinol. Metab. 317, E158-E171. doi: 10.1152/ajpendo.00521.2018

Okamoto, T., Morino, K., Ugi, S., Nakagawa, F., Lemecha, M., Ida, S., et al. (2019). Microbiome potentiates endurance exercise through intestinal acetate production. Am. J. Physiol. Endocrinol. Metab. 316, E956-E966. doi: 10.1152/ ajpendo.00510.2018

Oliveira, C. S., Pereira, H., Alves, S., Castro, L., Baltazar, F., Chaves, S. R., et al. (2015). Cathepsin D protects colorectal cancer cells from acetateinduced apoptosis through autophagy-independent degradation of damaged mitochondria. Cell Death Dis. 6:e1788. doi: 10.1038/cddis.2015.157

Petersen, L. M., Bautista, E. J., Nguyen, H., Hanson, B. M., Chen, L., Lek, S. H., et al. (2017). Community characteristics of the gut microbiomes of competitive cyclists. Microbiome 5:98. doi: 10.1186/s40168-017-0320-4

Pirozzi, C., Lama, A., Annunziata, C., Cavaliere, G., De Caro, C., Citraro, R., et al. (2020). Butyrate prevents valproate-induced liver injury: in vitro and in vivo evidence. FASEB J. 34, 676-690. doi: 10.1096/fj.201900927RR 
Przewłócka, K., Folwarski, M., Kaźmierczak-Siedlecka, K., Skonieczna-Żydecka, K., and Kaczor, J. J. (2020). Gut-Muscle AxisExists and may affect skeletal muscle adaptation to training. Nutrients 12:1451. doi: 10.3390/nu120 51451

Saunders, P. U., Garvican-Lewis, L. A., Schmidt, W. F., and Gore, C. J. (2013). Relationship between changes in haemoglobin mass and maximal oxygen uptake after hypoxic exposure. Br. J. Sports Med. 47(Suppl. 1), i26-i30. doi: 10.1136/bjsports-2013-092841

Scheiman, J., Luber, J. M., Chavkin, T. A., MacDonald, T., Tung, A., Pham, L. D., et al. (2019). Meta-omics analysis of elite athletes identifies a performanceenhancing microbe that functions via lactate metabolism. Nat. Med. 25, 11041109. doi: 10.1038/s41591-019-0485-4

Schloss, P. D., Westcott, S. L., Ryabin, T., Hall, J. R., Hartmann, M., Hollister, E. B., et al. (2009). Introducing mothur: open-source, platform-independent, community-supported software for describing and comparing microbial communities. Appl. Environ. Microbiol. 75, 7537-7541. doi: 10.1128/aem. 01541-09

Segata, N., Izard, J., Waldron, L., Gevers, D., Miropolsky, L., Garrett, W. S., et al. (2011). Metagenomic biomarker discovery and explanation. Genome Biol. 12:R60. doi: 10.1186/gb-2011-12-6-r60

Siebenmann, C., and Dempsey, J. A. (2020). Hypoxic training is not beneficial in elite athletes. Med. Sci. Sports Exerc. 52, 519-522. doi: 10.1249/mss. 0000000000002141

Smith, P. M., Howitt, M. R., Panikov, N., Michaud, M., Gallini, C. A., Bohlooly, Y. M., et al. (2013). The microbial metabolites, short-chain fatty acids, regulate colonic Treg cell homeostasis. Science 341, 569-573. doi: 10.1126/science. 1241165

Sun, G., Zhang, H., Wei, Q., Zhao, C., Yang, X., Wu, X., et al. (2019). Comparative Analyses of Fecal Microbiota in European Mouflon (Ovis orientalis musimon) and Blue Sheep (Pseudois nayaur) Living at Low or High Altitudes. Front. Microbiol. 10:1735. doi: 10.3389/fmicb.2019.01735

Suzuki, T. A., Martins, F. M., and Nachman, M. W. (2019). Altitudinal variation of the gut microbiota in wild house mice. Mol. Ecol. 28, 2378-2390. doi: 10.1111/ mec. 14905

Wang, L., Yang, S., Yan, L., Wei, H., Wang, J., Yu, S., et al. (2019). Hypoxia preconditioning promotes endurance exercise capacity of mice by activating skeletal muscle Nrf2. J. Appl. Physiol. 127, 1267-1277. doi: 10.1152/japplphysiol. 00347.2019

Woolcott, O. O., Ader, M., and Bergman, R. N. (2015). Glucose homeostasis during short-term and prolonged exposure to high altitudes. Endocr. Rev. 36, 149-173. doi: 10.1210/er.2014-1063

Zeng, Z., He, X., Li, F., Zhang, Y., Huang, Z., Wang, Y., et al. (2021). Probiotic Properties of Bacillus proteolyticus Isolated From Tibetan Yaks, China. Front. Microbiol. 12:649207. doi: 10.3389/fmicb.2021.649207

Zhao, G., Nyman, M., and Jönsson, J. A. (2006). Rapid determination of shortchain fatty acids in colonic contents and faeces of humans and rats by acidified water-extraction and direct-injection gas chromatography. Biomed. Chromatogr. 20, 674-682. doi: 10.1002/bmc.580

Zhao, X., Zhang, Z., Hu, B., Huang, W., Yuan, C., and Zou, L. (2018). Response of gut microbiota to metabolite changes induced by endurance exercise. Front. Microbiol. 9:765. doi: 10.3389/fmicb.2018.00765

Zhong, F., Wen, X., Yang, M., Lai, H. Y., Momma, H., Cheng, L., et al. (2021). Effect of an 8-week exercise training on gut microbiota in physically inactive older women. Int. J. Sports Med. 42, 610-623. doi: 10.1055/a-1301-7011

Conflict of Interest: The authors declare that the research was conducted in the absence of any commercial or financial relationships that could be construed as a potential conflict of interest.

Publisher's Note: All claims expressed in this article are solely those of the authors and do not necessarily represent those of their affiliated organizations, or those of the publisher, the editors and the reviewers. Any product that may be evaluated in this article, or claim that may be made by its manufacturer, is not guaranteed or endorsed by the publisher.

Copyright (c) 2022 Huang, Li, Zhou, Deng, Zhang, Yi, Zhu, Zhu and Mi. This is an open-access article distributed under the terms of the Creative Commons Attribution License (CC BY). The use, distribution or reproduction in other forums is permitted, provided the original author(s) and the copyright owner(s) are credited and that the original publication in this journal is cited, in accordance with accepted academic practice. No use, distribution or reproduction is permitted which does not comply with these terms. 